ARTICLE

\title{
Robust coherent control of solid-state spin qubits using anti-Stokes excitation
}

\author{
Jun-Feng Wang (1) 1,2, Fei-Fei Yan ${ }^{1,2}$, Qiang Li,2, Zheng-Hao Liu (1) 1,2, Jin-Ming Cui1,2, Zhao-Di Liu ${ }^{1,2}$, \\ Adam Gali (i) ${ }^{3,4 凶}$, Jin-Shi Xu (1D) ${ }^{1,2 凶}$, Chuan-Feng Li (iD ${ }^{1,2 凶}$ \& Guang-Can Guo ${ }^{1,2}$
}

Optically addressable solid-state color center spin qubits have become important platforms for quantum information processing, quantum networks and quantum sensing. The readout of color center spin states with optically detected magnetic resonance (ODMR) technology is traditionally based on Stokes excitation, where the energy of the exciting laser is higher than that of the emission photons. Here, we investigate an unconventional approach using antiStokes excitation to detect the ODMR signal of silicon vacancy defect spin in silicon carbide, where the exciting laser has lower energy than the emitted photons. Laser power, microwave power and temperature dependence of the anti-Stokes excited ODMR are systematically studied, in which the behavior of ODMR contrast and linewidth is shown to be similar to that of Stokes excitation. However, the ODMR contrast is several times that of the Stokes excitation. Coherent control of silicon vacancy spin under anti-Stokes excitation is then realized at room temperature. The spin coherence properties are the same as those of Stokes excitation, but with a signal contrast that is around three times greater. To illustrate the enhanced spin readout contrast under anti-Stokes excitation, we also provide a theoretical model. The experiments demonstrate that the current anti-Stokes excitation ODMR approach has promising applications in quantum information processing and quantum sensing.

\footnotetext{
${ }^{1}$ CAS Key Laboratory of Quantum Information, University of Science and Technology of China, Hefei, Anhui, People's Republic of China. ${ }^{2}$ CAS Center for Excellence in Quantum Information and Quantum Physics, University of Science and Technology of China, Hefei, Anhui, People's Republic of China.

${ }^{3}$ Department of Atomic Physics, Budapest University of Technology and Economics, Budapest, Hungary. ${ }^{4}$ Wigner Research Centre for Physics,

Budapest, Hungary. ${ }^{凶}$ email: gali.adam@wigner.hu; jsxu@ustc.edu.cn; cfli@ustc.edu.cn
} 
C olor centers in wide-bandgap semiconductors have been widely used in quantum networks ${ }^{1-5}$, quantum information processing ${ }^{6-10}$, and quantum sensing ${ }^{11-17}$ owing to their stable fluorescence properties and long spin coherence time even at room temperature ${ }^{10,13}$. Spins of color centers can be polarized by laser and manipulated by microwave (MW), allowing a readout approach operating on spin-dependent fluorescence detection ${ }^{1-10}$. Optically detected magnetic resonance (ODMR) technology can be used on this basis to detect the spin state, which is the foundation for applications of quantum technology ${ }^{1-17}$. However, previously, almost all ODMR readout protocols have used conventional Stokes excitation, in which the wavelength of the excitation laser is shorter than the zero phonon lines (ZPLs) of the color centers. Investigation of additional spin readout protocols could significantly extend the scope of solidstate quantum technologies. For example, photocurrent detection of magnetic resonance (PDMR) has been demonstrated and implemented in both nitrogen-vacancy (NV) centers in diamond and defects in silicon carbide (SiC) based on spin-dependent ionization dynamics, which benefits device integration ${ }^{18,19}$. However, PDMR usually requires complex doping and nanofabrication technologies, and the output signal has to be increased $^{18,19}$.

On the other hand, the anti-Stokes (AS) excitation process, in which the wavelength of the exciting laser is longer than that of the emitted photons, has been observed in quantum dots, dye molecules, and rare-earth doped crystals suited for applications, including Raman spectroscopy and laser cooling $20,21$. The mechanisms of AS excitation have been clarified, such as multiphoton absorption, phonon absorption, and Auger recombination ${ }^{20,21}$. Most recently, it has been also observed in the color centers of diamond and defects in hexagonal boron nitride $(\mathrm{hBN})^{20,21}$. These were shown to be helpful in alloptical temperature sensing and manipulating emissions of quantum emitters ${ }^{20,21}$. However, there are still no published studies on ODMR detection and coherent manipulation of solid-state spin states under AS excitation. The realization of spin readout with AS excitation would broaden the boundary of quantum technologies based on solid-state spin qubits.

In recent years, color centers in $\mathrm{SiC}$ have been studied as promising candidates for bright single photon sources ${ }^{22,23}$ and spin qubits $^{8-10}$. In contrast to diamond, $\mathrm{SiC}$ is a technology-friendly semiconductor with mature micro-nano fabrication and inch-scale growth technologies. Optically addressable spin qubits, including silicon vacancy $\left(\mathrm{V}_{\mathrm{Si}}\right)$ defects ${ }^{4,10}$, divacancy ${ }^{8,9}, \mathrm{NV}$ centers ${ }^{24-26}$, in $\mathrm{SiC}$ have drawn much attention due to their near-infrared and even telecom-ranged fluorescence and long spin coherence time $e^{4,9,10,24-26}$. These superior characteristics make them extensively applicable to quantum information processing ${ }^{8-10}$, spin-photon interfaces 4,5 , and quantum metrology, such as magnetic field ${ }^{15}$, electric field ${ }^{16}$, and temperature ${ }^{17}$.

In this work, we realize the coherent control of $\mathrm{V}_{\mathrm{Si}}$ defect ensemble spins in $4 \mathrm{H}-\mathrm{SiC}$ under AS excitation at room temperature. First, AS photoluminescence (PL) is investigated as a function of AS exciting laser power, temperature, and duration times, showing that AS PL is caused by the phonon-assisted single photon absorption process. On this basis, the Stokes and AS excited ODMR spectra are obtained for different excitation laser power, MW power, and temperature, which are shown to follow similar behavior. We then measured the external magnetic fielddependent Stokes and AS ODMR, both of which exhibit the same Zeeman splitting phenomena. Finally, a comparison of the free induction decay and spin echo of $\mathrm{V}_{\mathrm{Si}}$ defects under Stokes and AS excitation reveals that AS excitation does not affect the coherent properties of $\mathrm{V}_{\mathrm{Si}}$ defects. Hence, the demonstrated AS excited
ODMR approach shows potential for quantum information processing and quantum sensing.

\section{Results}

$\mathrm{V}_{\mathrm{Si}}$ defects are point defects containing a silicon vacancy, which exist in $4 \mathrm{H}-\mathrm{SiC}$ and $6 \mathrm{H}-\mathrm{SiC}$. In $4 \mathrm{H}-\mathrm{SiC}$, two forms of $\mathrm{V}_{\mathrm{Si}}$ defects exist due to discrepancies in the silicon vacancy lattice: V1 and V2 centers have corresponding ZPLs of 861 and $915 \mathrm{~nm}$, respectively. Since the spin of the V2 center can be manipulated at room temperature, we focus only on the V2 center of the $\mathrm{V}_{\mathrm{Si}}$ defect, which features a $S=3 / 2$ spin quartet and $70 \mathrm{MHz}$ ground state zero field splitting (ZFS). A home-made confocal system combined with a MW system is implemented to excite and manipulate the $V_{\mathrm{Si}}$ defect (see details in "Methods"). Lasers with wavelengths of 720 and $1030 \mathrm{~nm}$ are used for the Stokes and AS excitation of $\mathrm{V}_{\mathrm{Si}}$ defects, respectively. A band-pass filter with a transmitting wavelength of $850-1000 \mathrm{~nm}$ is used in the confocal set-up. In the experiment, we use a $4 \mathrm{H}-\mathrm{SiC}$ sample with highdensity $\mathrm{V}_{\mathrm{Si}}$ defect ensembles ${ }^{27}$ (see details in "Methods").

The proposed energy model for PL and optically induced spin polarization of the Stokes and AS excitation of $\mathrm{V}_{\mathrm{Si}}$ defect in $4 \mathrm{H}$ $\mathrm{SiC}$ is shown in Fig. $1 \mathrm{a}^{20,21,28-31}$. The defect has $S=3 / 2$ ground and optically accessible excited state with $m_{\mathrm{s}}= \pm 1 / 2$ and $m_{\mathrm{s}}=$ $\pm 3 / 2$ spin manifolds. The ground state and the lowest energy $S=3 / 2$ excited state have ${ }^{4} \mathrm{~A}_{2}$ symmetry. In this case, we use a five-level scheme where the series of doublet states is substituted by a single effective doublet state (state 5 ). The electron may scatter from the excited state spin manifolds (states 3 and 4 , i.e., $m_{\mathrm{s}}= \pm 1 / 2$ and $m_{\mathrm{s}}= \pm 3 / 2$, respectively) to this state or can scatter from this state to the ground state spin manifolds (states 1 and 2, i.e., $m_{\mathrm{s}}= \pm 1 / 2$ and $m_{\mathrm{s}}= \pm 3 / 2$, respectively). For the usual Stokes excitation (Fig. 1a left part), both the 1 and 2 ground states transmit to the excited states phonon sideband under the laser excitation. Generally, the simple phonon relaxation and excitation processes should be spin-conserving (only contain spin-independent operators). By phonon relaxation, they then rapidly thermalize to the 3 and 4 states respectively, followed by the spin-preserving radiative transition to the ground states (see Supplementary Note 1 for more details). In the AS excitation (Fig. 1a right part), the probability of going from the ground state manifold to the excited state manifold is less than that in the Stokes excitation, since it involves the phonon excitation in the former process. For both the Stokes and AS excitations, we assume that the non-radiative intersystem crossover to the metastable doublet state occurs primarily from state 3 , then they polarized to state $1^{30}$.

We then investigated the AS PL of $\mathrm{V}_{\mathrm{Si}}$ defects in $4 \mathrm{H}-\mathrm{SiC}$. The PL spectra of the $\mathrm{V}_{\mathrm{Si}}$ defects under Stokes $(720 \mathrm{~nm}$, blue) and AS excitation is seen in Fig. $1 \mathrm{~b}(1030 \mathrm{~nm}$, red). Compared with the Stokes PL, the AS excited PL has a small red shift, which is consistent with previous results of NV centers in diamond ${ }^{20}$. To recognize the origin of the $\mathrm{AS} \mathrm{PL}$, we measure counts as a function of laser power $(P)$ of the Stokes and two AS $(1030 \mathrm{~nm}$ and $1064 \mathrm{~nm}$ ) excitations (Fig. 1c). The Stokes PL is fitted using the function $I(P)=I_{\mathrm{s}} /\left(1+P_{0} / P\right)$, where the maximal count $I_{\mathrm{s}}$ is $2350 \mathrm{kcps}$ and the saturation power $P_{0}$ is $4.3 \mathrm{~mW}$, respectively. However, both of the AS excited counts follow a linear fit, and the counts with $1030 \mathrm{~nm}$ laser excitation are 5.5 times larger than that of the $1064 \mathrm{~nm}$ laser excitation. The linear laser power-dependent behavior indicates that lower energy single photons rather than double photons are absorbed in the AS excitation process. In comparison, the efficiency of AS excitation becomes higher when the excitation wavelength is closer to the ZPL, which is consistent with that of color centers in diamond $\mathrm{d}^{20,28}$ and defects in $\mathrm{hBN}^{21}$. 

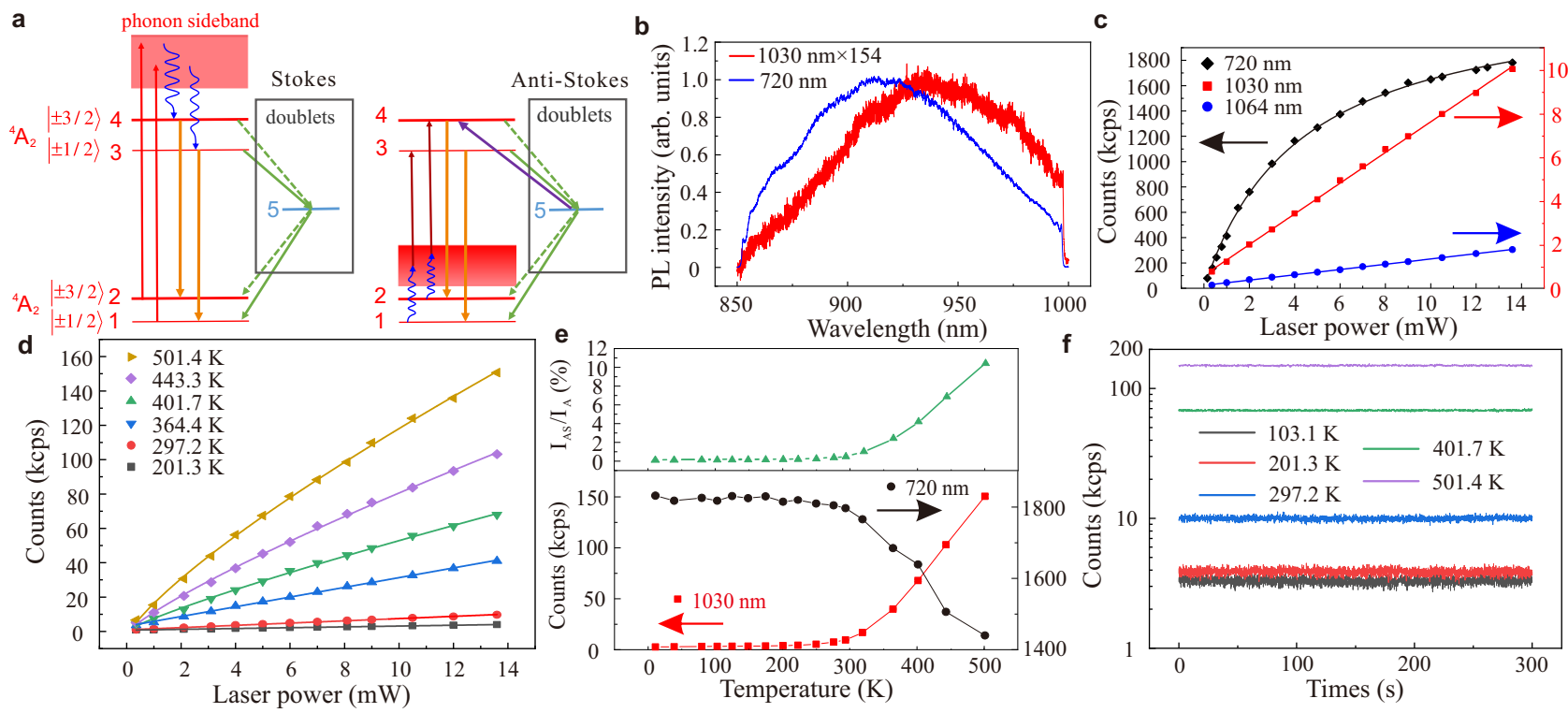

Fig. 1 The AS excitation model and the AS PL of $\mathbf{V}_{\mathbf{S i}}$ defects in $\mathbf{4 H - S i C}$. a Excitation schemes of $\mathbf{V}_{\mathrm{Si}}$ defects under Stokes excitation (left part) and antiStokes excitation (right part). The red (dark brown) arrows represent optical excitation from the ground state (labels 1 and 2) toward the excited states (labels 3 and 4) and the yellow arrows represent the associated spin-preserved radiative decay. The green arrows represent intersystem crossing (ISC) routes where dotted arrows mean weaker transitions than those represented by straight arrows. Blue wavy arrows show the phonon-related decay and excitation routes that are temperature dependent. The purple arrow represents a spin-flipping effective optical excitation from a metastable doublet state toward, selectively, to state 4 . This is the key process in increasing the ODMR contrast upon anti-Stokes excitation, which is discussed in detail in the part of theoretical analysis. The energy differences are not linearly scaling for the sake of clarity. $\mathbf{b}$ The PL spectra of $\mathrm{V}_{\mathrm{Si}}$ defects under Stokes (blue) and AS (red) excitation at room temperature, respectively. The units for both cases are equal, and the Stokes excited PL spectrum intensity is approximately 154 times larger than that of anti-Stokes. c The counts of $\mathrm{V}_{\mathrm{Si}}$ defects as a function of laser power under Stokes ( $720 \mathrm{~nm}$, black) and two different AS (1030 nm, red; $1064 \mathrm{~nm}$, blue) excitations at room temperature, respectively. The corresponding lines represent the fits. $\mathbf{d}$ The counts of $V_{S i}$ defects with respect to the laser power under AS excitation $(1030 \mathrm{~nm}$ ) at various temperatures. e The bottom panel displays the maximal counts with $13.6 \mathrm{~mW}$ Stokes (black) and AS (red) excitations as a function of temperature, respectively. The top panel shows the corresponding temperature-dependent ratio of AS to Stokes counts. $\mathbf{f}$ The AS PL time trace at five different temperatures with a time bin of $100 \mathrm{~ms}$.

The temperature-dependent AS excitation is investigated to further validate that the AS excitation involves a phonon-assisted process. The AS count scales linearly with the exciting laser power for one-photon absorption, while it scales quadratically for twophoton absorption $20,21,28$. Figure 1d presents the AS excited counts with respect to laser power at different temperatures, where the counts increase quickly as the temperature rises. At temperatures $<365 \mathrm{~K}$, the counts linearly increase with laser power, which indicates that the AS PL is due to the phononassisted single low-energy photon absorption process (see Supplementary Note 2). In comparison, at temperatures $>365 \mathrm{~K}$, the data were fitted using a function of $b P^{n}$ where the power index $n$ decreases from 0.85 to 0.78 as the temperature increases from 401.7 to $501.4 \mathrm{~K}$ and $b$ represents the temperature-dependent increasing parameter ${ }^{21,28}$. The decrease of the power index $n$ might be attributed to the reduction in efficiency of the phononassisted single photon absorption process.

To quantify the AS excitation efficiency, we use the ratio $I_{\mathrm{AS}} / I_{\mathrm{S}}$ of AS $\left(I_{\mathrm{AS}}\right)$ to Stokes $\left(I_{\mathrm{S}}\right)$ excited counts ${ }^{20}$. A summary of the Stokes and AS excited counts as a function of the temperature is plotted in the lower panel in Fig. 1e. The Stokes excited count $I_{S}$ is almost constant for temperatures varying from 11 to $300 \mathrm{~K}$ but declines rapidly as the temperature rises to $500 \mathrm{~K}$. In comparison, when the temperature rises from 11 to $300 \mathrm{~K}$, the AS excitation counts $I_{\mathrm{AS}}$ increase slowly and then increase rapidly as the temperature rises to $500 \mathrm{~K}$. The ratio $I_{\mathrm{AS}} / I_{\mathrm{S}}$ increases slowly as the temperature increase to $300 \mathrm{~K}$, which is shown in the upper panel in Fig. 1e. At room temperature, $I_{\mathrm{AS}} / I_{\mathrm{S}}$ is around $0.57 \%$, which is approximately 50 times the ratio for $\mathrm{NV}$ centers in diamond ${ }^{20}$. $I_{\mathrm{AS}} / I_{\mathrm{S}}$ can be further increased using a shorter wavelength of AS excited laser 20,21 . In addition, $I_{\mathrm{AS}} / I_{\mathrm{S}}$ increases to $10.4 \%$ at $500 \mathrm{~K}$, which is comparable with the silicon vacancy and germanium vacancy in diamond at room temperature ${ }^{20}$. The dramatic increase in the behavior of $I_{\mathrm{AS}} / I_{\mathrm{S}}$ at elevated temperatures will be useful for all-optical high temperature sensing ${ }^{20}$. Combined with $\mathrm{SiC}$ nano-particles ${ }^{32}$, nanoscale temperature sensors usable in living cells and microelectronic systems can be constructed. Through manufacturing photonics structures such as solid immersion lenses ${ }^{10}$ and so on, it is possible to realize AS excitation of a single $\mathrm{V}_{\mathrm{Si}}$ defect ${ }^{20}$. We further investigated the stability of PL under AS excitation at various temperatures. Figure if displays the results with five representative temperatures, with all of the counts stable for these temperatures. We also note that the low PL photon counts for the AS approach would limit the application by employing only single emitters, while the quantum-sensing sensitivity can be dramatically increased by a factor of $\sqrt{N}$, where $N$ is the number of the color centers ${ }^{33}$. Ensemble color centers would be useful for high-sensitivity quantum sensing.

On the basis of stable phonon-assisted single photon emissioninduced AS PL, we investigate AS excited ODMR of $\mathrm{V}_{\mathrm{Si}}$ defect ensemble and compare it with Stokes excited ODMR. Figure 2a shows two representative Stokes and AS excited ODMR spectra at different laser powers with the same MW power, which is characterized by the Rabi frequency and the value is read as $1.03 \mathrm{MHz}$. The contrast of both the Stokes and AS excited ODMR at $4 \mathrm{~mW}$ laser power is slightly larger than at $10 \mathrm{~mW}$. In addition, under AS excitation, the contrast is around three times that of Stokes excitation. Comparisons of ODMR contrast and linewidth as a function of laser power under Stokes and AS excitation are 

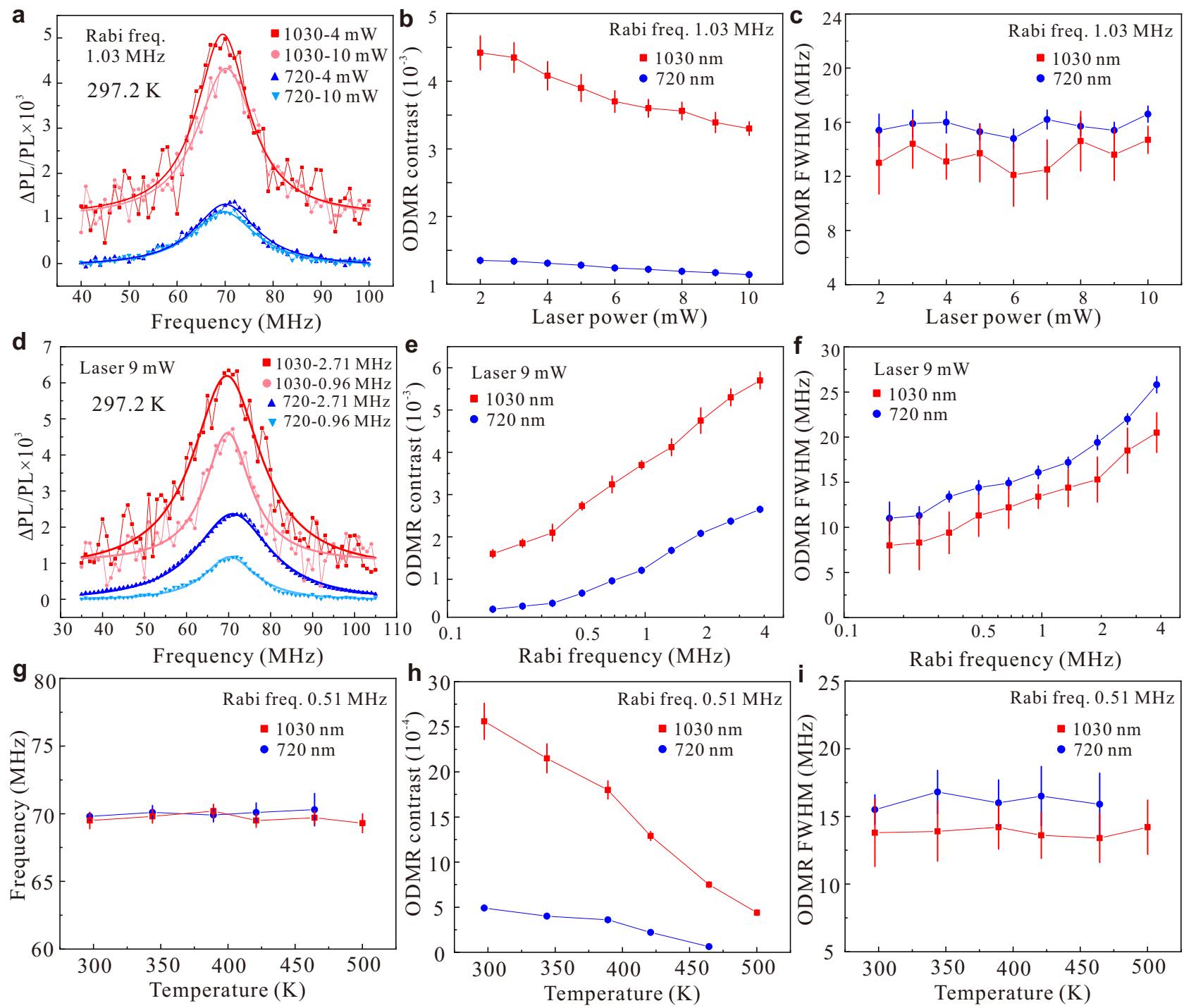

Fig. 2 The ODMR spectra of $\mathbf{V}_{\mathbf{S i}}$ defects under Stokes and AS excitation at zero external magnetic field. a Two representative Stokes and AS excited ODMR signals with different laser powers at room temperature (297.2 K). The lines are fitted using Lorentzian functions. b, c Comparison of Stokes and AS excited ODMR contrast and full width half maximum (FWHM) as a function of laser power, respectively. d Two representative Stokes and AS excited ODMR signals with different MW powers (Rabi frequencies) at room temperature (297.2 K). e, f Measurement of Stokes and AS excited ODMR contrast and FWHM as a function of Rabi frequency, respectively. $\mathbf{g}$ ZFS of $\mathrm{V}_{\mathrm{Si}}$ defects under Stokes and AS excitation as a function of temperature. $\mathbf{h}, \mathbf{i}$, Comparison of Stokes and AS excited ODMR contrast and FWHM with respect to the temperature. Error bars represent the standard deviations of the corresponding fittings.

presented in Fig. 2b, c, respectively. Both the ODMR contrast decreases as the laser power increases, and the AS ODMR contrast remains around three times larger than the Stokes one. Meanwhile, both ODMR linewidths stay almost the same as laser power increases, in line with previous results ${ }^{19}$. The mean AS excited ODMR linewidth is around $2.2 \pm 0.7 \mathrm{MHz}$ smaller than that of the Stokes excitation. For two different MW powers, representative Stokes and AS excited ODMR signals at the same laser power are shown in Fig. 2d. Both the ODMR contrast and linewidth at higher Rabi frequency are larger than that at lower frequency, and the AS excited ODMR contrast values are also much higher than the Stokes ones. However, the ODMR signalto-noise ratio (SNR) of the AS excitation is still low, which is due to the low AS excited counts and intrinsic low ODMR contrast of the silicon vacancy ${ }^{10,27}$. Unlike the Stokes excited counts that have smaller saturation power of the Stokes laser, the AS excited counts linearly increase as the AS exciting laser power. In the experiments, we can use a higher laser power $(>400 \mathrm{~mW})$ to significantly increase AS excited counts ${ }^{34}$. In addition, the thermally quenched methods will increase the intrinsic ODMR contrast by about ten times ${ }^{34}$. So, using the thermally quenched sample and higher AS exciting laser power, the ODMR SNR of the AS excitation will obviously increase ${ }^{34}$.

Figure 2e, f display a comparison of the ODMR contrast and linewidth under Stokes and AS excitation as a function of MW power. Both the ODMR contrast and linewidth increase as the MW power increases with similar behavior. In comparison, the AS excited ODMR contrast is much higher than that for Stokes excitation, while the linewidth is again lower than the Stokes excitation one. To understand this phenomenon, we assume that the AS excitation energy may excite the electron from a metastable doublet state, selectively, to state 4 , which represents a spinflipping effective optical excitation (see Fig. 1b). In this scenario, the brighter spin state is occupied in the quartet excited state 
manifold, which contributes to an enhanced AS excited ODMR contrast. We discuss the possible mechanism in detail in the theoretical part.

Since the AS excited PL counts increase rapidly as temperature increases, the temperature dependence of the AS excited ODMR signals has been investigated. Figure $2 \mathrm{~g}$ shows the resonance frequencies with respect to temperature. The half-integer spin $(S=3 / 2)$ of the silicon vacancy in $4 \mathrm{H}-\mathrm{SiC}$ makes it insensitive to fluctuations in strain, temperature, and electric field ${ }^{35}$. The resonance frequencies are almost the same under AS and Stokes excitation conditions (the mean difference is around $0.3 \pm$ $0.3 \mathrm{MHz}$ ). Different from the behavior of the divacancies in $\mathrm{SiC}^{17,36}$ and $\mathrm{NV}$ centers in diamond $\mathrm{d}^{37,38}$, both the values are constant as the temperature increases, which is similar to low temperature results ${ }^{39-41}$. The results show that ZFS is almost temperature independent (see Supplementary Note 2) ) $^{39-41}$, which is useful for temperature-independent quantum sensing such as magnetic field and electric field sensing in broad temperature ranges $^{39,41}$. A comparison of ODMR contrast and linewidth as a function of temperature is displayed in Fig. $2 \mathrm{~h}$, i, respectively. Although the contrast for both cases decreases as temperature increases, the AS excited ODMR contrast is approximately four times that of Stokes as the temperature rises from 297.2 to around $421 \mathrm{~K}$. Moreover, the AS excited ODMR signal can still be detected at $500 \mathrm{~K}$, while the Stokes excited ODMR signal is undetectable at the same experimental conditions. This property is useful for high-temperature quantum sensing, which is not only of interest in extending the scope of quantum sensing but also of practical applications in many important fields of nanotechnology ${ }^{42}$. Distinct to ODMR contrast, both linewidths are almost constant, with the AS excited ODMR linewidth also smaller than the Stokes excitation one. The results demonstrate that the AS excited ODMR signals are more robust than that of the Stokes excitation.

After optimizing the AS excited ODMR spectrum, we then studied its magnetic field-dependent behavior, which is the basis of quantum sensing. Under an external magnetic field, there are two dipole-allowed transitions $|-1 / 2\rangle \leftrightarrow|-3 / 2\rangle$ and $|1 / 2\rangle \leftrightarrow|3 / 2\rangle$, with corresponding transition frequencies of $\left|2 D-g \mu_{\mathrm{B}} B\right|$ and $\left|2 D+g \mu_{\mathrm{B}} B\right|$, respectively. The parameters $D, g$, $\mu_{\mathrm{B}}$, and $B$ represent ZFS, Lande $g$ factor, Bohr magneton, and external magnetic field, respectively. Figure 3a exhibits the Stokes and AS excited ODMR spectra for three different $c$-axis magnetic fields. For convenience, the laser powers for the Stoke and AS cases are set at 1 and $14 \mathrm{~mW}$, respectively, while the MW power is set to be the same. Both Stokes and AS excited ODMR spectra show the same splitting; however, contrast under AS excitation is approximately three times that of Stokes excitation. For example, at the magnetic field of $52 \mathrm{G}$, the Stokes and AS excited ODMR resonance frequencies are $77.4(77.8)$ and $214.1(213.8) \mathrm{MHz}$, respectively. A summary of both Stokes and AS excited ODMR resonance frequencies at various magnetic fields is shown in Fig. $3 \mathrm{~b}$. The blue lines are the calculated transition frequencies. All the measured resonance frequencies of Stokes and AS excited ODMR coincide and agree well with the calculated results. As with Stokes excited ODMR, the demonstrated AS excited ODMR technology can also be used for direct current magnetic field sensing ${ }^{15}$.

High-quality coherence properties of solid-state spin qubits are significant for quantum computing ${ }^{8-10}$ and high-sensitivity quantum sensing ${ }^{16,17}$. In view of this, we study the coherence properties of $\mathrm{V}_{\mathrm{Si}}$ defects under AS excitation. The experimental coherence control pulse sequences are the same for both the Stokes and AS excitations, which are also the same to the previous standard pulse sequences ${ }^{8,17,25}$ (see Supplementary Note 3). As shown in Fig. 4a, the Rabi oscillations under Stokes (blue) and AS (red) excitations are measured using a resonant frequency of $77.5 \mathrm{MHz}$ at $52 \mathrm{G}$ with standard Rabi pulse sequences at room temperature ${ }^{17,25}$. From the fit, the AS excited Rabi frequency is $8.01 \pm 0.09 \mathrm{MHz}$, which is the same as that of Stokes $(7.94 \pm$ $0.05 \mathrm{MHz}$ ). The contrast of the AS excited Rabi oscillation is around three times that of the Stokes results at the same time. Figure $4 \mathrm{~b}$ presents the calculated Rabi frequencies at different MW power under Stokes (blue) and AS (red) excitation, respectively. The frequencies are almost the same and linearly increase with the square root of the MW power. The results demonstrate that Rabi oscillation follows the same behavior under MW fields for both Stokes and AS excitations.

To measure the inhomogeneous spin-dephasing time under AS excitation, we employ the standard Ramsey pulse sequence. The comparison of Stokes (blue) and AS (red) excited Ramsey oscillations is presented in Fig. 4c. The spin dephasing time $T_{2}{ }^{*}$ for the AS (Stokes) excitation is $0.38 \pm 0.05 \mu \mathrm{s}(0.35 \pm 0.04 \mu \mathrm{s})$, respectively. The experiments show that the AS excitation method not only does not change the spin-dephasing time but also can increase the signal contrast by approximately three times. We subsequently investigate the AS excited spin coherence time using the spin echo pulse sequence. The AS excited coherence time $T_{2}$ is found to be $7.1 \pm 0.6 \mu \mathrm{s}$, which is also consistent with the Stokes value of $6.3 \pm 0.4 \mu \mathrm{s}$. As with dephasing time, spin coherence time
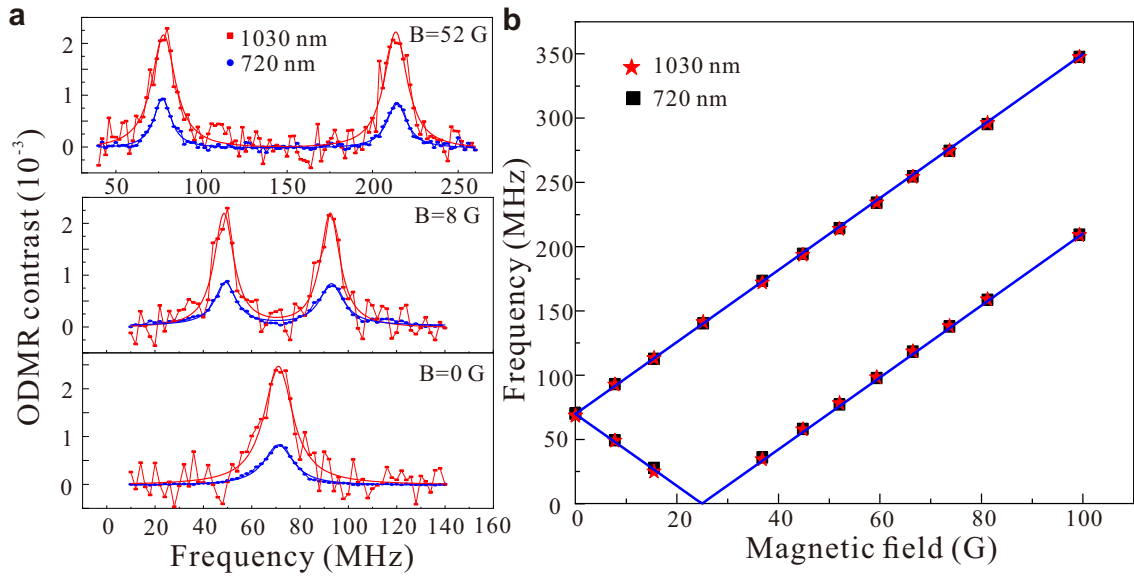

Fig. 3 Stokes and AS excited ODMR spectra of $\mathbf{V}_{\mathbf{S i}}$ defects for different external magnetic fields at room temperature. a Three representative Stokes (blue) and $\mathrm{AS}$ excited (red) ODMR spectra of $\mathrm{V}_{\mathrm{Si}}$ defects for the same Rabi frequency $(0.8 \mathrm{MHz})$ at different c-axis external magnetic fields. $\mathbf{b}$ Stokes (black) and AS excited (red) ODMR resonance frequencies as a function of the $c$-axis magnetic field. 

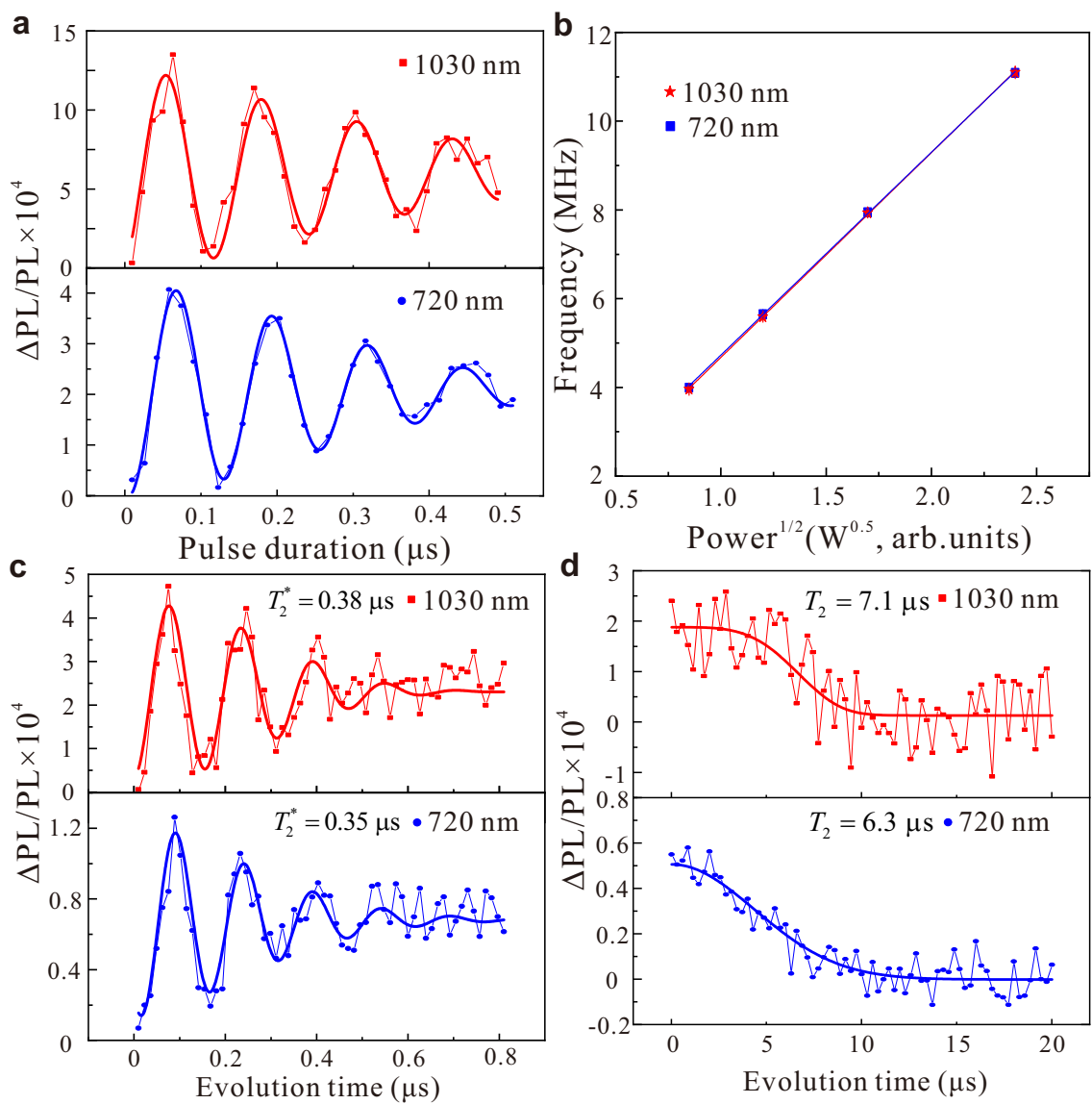

Fig. 4 Coherent control of $\mathbf{V}_{\mathbf{S i}}$ defects at room temperature under Stokes and AS excitation. a Measurement of Rabi oscillation under Stokes (blue) and AS (red) excitation at the same MW power at $52 \mathrm{G}$. b Measured Rabi frequencies as a function of MW power under Stokes (blue) and AS (red) excitation. The lines are the linear fits to the data and match with each other. c, d Comparison of the Ramsey and spin echo measurements under Stokes (blue) and AS (red) excitations, respectively.

is not affected by the AS excitation method. The comparison of coherent control under Stokes and AS excitations demonstrates that the AS excitation technology does not affect the spin coherence properties. Moreover, it can also increase the signal contrast about three times, which is beneficial for experiments. In addition, coherent control of single $\mathrm{V}_{\mathrm{Si}}$ defects may also be realized using photon structures at a higher temperature.

Theoretical analysis on the ODMR contrast. To make the explanation for the increased ODMR contrast for the AS approach as compared to the Stokes approach more clear, we first discuss the relationship of the continuous wave (CW) ODMR contrast between the decay rates with the energy levels shown in Figs. 1a and 5 that we took from first principles calculations ${ }^{43}$. We label the states with $m_{\mathrm{s}}$ manifolds by numbers (see also Supplementary Note 1). For the case of Stokes excitation, radiative decay occurs from state 3 to state 1 and from state 4 to state 2 with the rate of $r_{\mathrm{d}}$. The corresponding intersystem crossing (ISC) rates between states $i$ and $j$ are labeled by $r_{i j}$. The occupation of $i$ level is labeled by $n_{i}$. The total intensity of fluorescence $I=I_{ \pm 1 / 2}+I_{ \pm 3 / 2}$, where

$$
I_{ \pm 1 / 2}=\frac{n_{1} c_{1} r_{\mathrm{d}}}{r_{\mathrm{d}}+r_{35}}
$$

and

$$
I_{ \pm 3 / 2}=\frac{n_{2} c_{1} r_{\mathrm{d}}}{r_{\mathrm{d}}+r_{45}}
$$

where $c_{1}$ is the rate of photo-excitation. In the weak illumination limit, the electron dominantly occupies the ground state spin manifolds, i.e., $n_{1}+n_{2} \approx 1$, where the spin-polarization $P$ between the states can be expressed as $n_{1}-n_{2} \approx P$ because $m_{\mathrm{s}}= \pm 1 / 2$ spin state is spin-polarized according to previous measurements ${ }^{44}$. Upon illumination with a strength of $c_{1}<r_{\mathrm{d}}$, the CW ODMR contrast $C$ may be expressed as

$$
C=\frac{I(P=0)}{I(P=1)}-1
$$

where Eq. (3) together with Eqs. (1) and (2) simplifies to

$$
C=0.5 \frac{r_{35}-r_{45}}{r_{\mathrm{d}}+r_{45}}
$$

A small positive ODMR contrast is developed by the condition of $r_{35}>r_{45}$, i.e., a faster ISC process from the $m_{\mathrm{s}}= \pm 1 / 2$ manifold than from the $m_{s}= \pm 3 / 2$ manifold toward the doublet states, which is observed in the experiments.

In the AS excitation, we suppose the simple phonon relaxation and excitation processes should be spin-conserving. As shown in Fig. 1a, the probability to get from the quartet ground state manifold to the quartet excited state manifold is less than that in the Stokes excitation (labeled as $c_{1}$ ) because it involves the phonon excitation in the former process. Once the electron decays from the quartet excited state to the lowest energy doublet state, which is a metastable state with relatively long lifetime, the CW laser used for AS excitation may excite the electron from the metastable doublet state, selectively, to state $4\left(m_{\mathrm{s}}= \pm 3 / 2\right.$ spin state). The process is represented by a purple arrow in Figs. 1a and $5 b$, which competes with the decay of the electron from the 

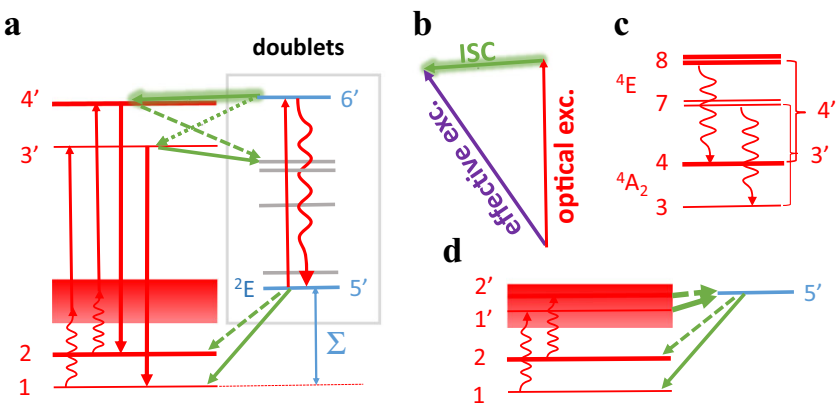

Fig. 5 Anti-Stokes excitation scheme of $\mathbf{V}_{\mathbf{S i}}$ defects. a Energy diagram of the quartet (red horizontal lines) and doublets. The position of the doublets (blue and gray horizontal lines) is not exactly known. The energy differences are not linearly scaled for the sake of clarity, which includes the ionic relaxations in each multiplet (ZPL energies). The shaded area is the phonon sideband of states 1 and 2 . The lowest energy doublet $(2 \mathrm{E})$ is shown among the other doublets. $\Sigma$ is the energy difference between the ground state manifold and the ${ }^{2} \mathrm{E}$ level (state $5^{\prime}$ ). A predominant spin-selective intersystem crossing (ISC) occurs between a resonant doublet state and 4' ( $m_{\mathrm{s}}= \pm 3 / 2$ manifolds). $\mathbf{b}$ The optical excitation (red arrow) between the doublets ${ }^{2} E$ (state $5^{\prime}$ ) and the highest doublet close to resonance with the quartet levels (state $6^{\prime}$ ) followed by a fast ISC (shaded green arrow) toward the $m_{\mathrm{s}}= \pm 3 / 2$ states due to spin-orbit coupling leads to an effective spinflipping optical excitation (purple arrow). There might be a weaker ISC toward the $m_{\mathrm{s}}= \pm 1 / 2$ states (green dotted arrow) too. c The nature of 3' and $4^{\prime}$ states is depicted, which are the phonon coupled $m_{\mathrm{s}}= \pm 3 / 2$ ( 3 and 7) and $m_{s}= \pm 1 / 2$ states ( 4 and 8 ) from the ${ }^{4} A_{2}$ and ${ }^{4} E$ quartet excited states, respectively. $\mathbf{d}$ The high energy vibronic states $1^{\prime}$ and $2^{\prime}$ of states 1 and 2, respectively. ISC with enhanced rates may occur from states $1^{\prime}$ and $2^{\prime}$ to state $5^{\prime}$ beside the optical excitation toward states $3^{\prime}$ and $4^{\prime}$, respectively.

metastable doublet state to the quartet ground state by ISC. In this scenario, the brighter spin state can be increasingly populated in the quartet excited state manifold (state 4), which leads to an enhanced CW ODMR readout contrast (see also Supplementary Note 1).

The effective spin-flipping optical excitation may be explained by a relatively strong optical excitation between the doublets followed by a spin-selective ISC toward the $m_{\mathrm{s}}= \pm 3 / 2$ spin states (see Fig. 5b). The refined model is depicted in Fig. 5a. According to first principles calculations beyond density functional theory ${ }^{43}$, the lowest energy doublet level $\left({ }^{2} \mathrm{E}\right)$ is at about $\Sigma=0.15 \mathrm{eV}$ above the quartet ground state's level, which is explicitly given and labeled as state 5'. The AS excitation energy is able to promote this electron to state 6', which is higher energy doublet state and quasi-degenerate with the quartet levels, depicted explicitly in the series of other doublets. We assume that there is a dominant relaxation path from state $6^{\prime}$ toward the $m_{\mathrm{s}}= \pm 3 / 2$ spin states, which is further considered in our analysis. The exact nature of state 6 is not known at the moment from literature ${ }^{43}$ but may be associated with a doublet ${ }^{2} \mathrm{E}$ counterpart of the ${ }^{4} \mathrm{E}$ quartet (see the discussion in Supplementary Note 1). The ${ }^{4} \mathrm{E}$ state is the second optically allowed excited state that is separated from the ${ }^{4} \mathrm{~A}_{2}$ state by about $20 \mathrm{meV}$, and they are strongly coupled by phonons $\mathrm{s}^{45}$ (see Fig. 5c). We label the corresponding spin states of ${ }^{4} \mathrm{E}$ manifold as 7 and 8 . As the phonon coupling is strong and the phonon decay process is considered to be rapid between states 8 and 4 as well as states 7 and 3 (c.f., Figs. 1a and 5c), the electron then decays either from state 4 or state 3 toward the lower energy doublets by ISC or toward the ground state manifold by emitting photons. Therefore, the corresponding joint states are labeled as 4' $\left(m_{\mathrm{s}}= \pm 3 / 2\right.$ states $)$ and 3' ( $m_{\mathrm{s}}= \pm 1 / 2$ states $)$ in Fig. $5 \mathrm{a}$.

The CW ODMR contrast changes because the optically excited bright $m_{\mathrm{s}}= \pm 3 / 2$ spin state can be occupied from the doublets in
AS excitation as a new source of the occupation of that state with respect to that of the Stokes excitation. We show this effect briefly by calculating the CW ODMR contrast upon AS illumination with the model sketched in Fig. 5. For the sake of simplicity, the prime labels are omitted in the following equations that describe the possible decay and excitation routes. $I_{ \pm 1 / 2}$ in Eq. (1) does not change. However, $I_{ \pm 3 / 2}$ modifies to

$$
I_{ \pm 3 / 2}=\frac{n_{2} c_{1} r_{\mathrm{d}}+n_{6} r_{64} r_{\mathrm{d}}}{r_{\mathrm{d}}+r_{45}}
$$

where $r_{64}$ is the ISC rate from state 6 to state 4 '. The occupation of level 6' $\left(n_{6}\right)$ depends on the occupation of level $5^{\prime}\left(n_{5}\right)$ and the strength of optical excitation toward state $6^{\prime}\left(c_{56}\right)$, which should be much higher than $c_{1}$ in the AS excitation because it does not require the absorption of phonons (see also Supplementary Note 1). The occupation of levels 5 ' and 6 ' in the steady state may be given as

$$
n_{5}=\frac{n_{3} r_{35}+n_{4} r_{45}}{r_{51}+r_{52}+c_{56}}
$$

and

$$
n_{6}=\frac{n_{5} c_{56}}{r_{63}+r_{65}}
$$

By assuming that $r_{64}$ is large (fast ISC because of the resonance conditions and sufficiently large spin-orbit interaction ${ }^{45}$, one can again assume $n_{1}+n_{2} \approx 1$ and $n_{1}-n_{2} \approx P$ similarly to the case of Stokes excitation. By combining Eqs. (1), (6), and (7), one can express Eq. (5) as a function of $n_{1}$ and $n_{2}$, where the contrast is again defined by Eq. (3). Finally, the calculated CW ODMR contrast of AS excitation will be larger than that for Stokes excitation because $I(P=1 / 2)$ is unchanged but $I(P=3 / 2)$ increases because of the increase in $I_{ \pm 3 / 2}$. We note that this basic result is not altered by considering a weak scattering from the state 6' toward 3' $\left(r_{63}<r_{64}\right)$, which is ignored now for the sake of clarity.

We further note that an additional effect may contribute to the enhanced CW ODMR contrast upon AS excitation (see Fig. 5d). Because the system is spin-polarized to state 1 , it is plausible to assume that $r_{51}>r_{52}$, i.e., continuous green arrow vs. dotted green arrow in Fig. 5a. In the AS excitation of the ground state manifold, a photon is absorbed first, thus we arrive at state 1' from state 1 and at state 2 ' from state 2 . If the intensity of the pumping laser is not sufficiently high, then ISC from states 1' and 2 ' to state 5 ' may conquer with the optical excitation from states $1^{\prime}$ ' and 2' to states 3 ' and 4', respectively, where $r_{51}>r_{52}$, because of the assumption of $r_{51}>r_{52}$ and the spin-orbit couplingresponsible for ISC between the doublet and the quartet-is not affected directly by phonons (see Supplementary Note 1 for details). As a consequence, the occupation of state 3 ' is effectively depleted with respect to that of state 4 , and thus $I_{ \pm 1 / 2}$ effectively decreases over $I_{ \pm 3 / 2}$ with respect to the case of Stokes excitation. This effect may increase the CW ODMR contrast of AS excitation compared to that of Stokes excitation.

\section{Discussion}

In conclusion, we realized the coherent control of $\mathrm{V}_{\mathrm{Si}}$ defect spin ensembles in $4 \mathrm{H}-\mathrm{SiC}$ under AS excitation at room temperature. Through investigation of the AS PL dependence on laser power and temperature, we demonstrate that the AS PL is induced by the phonon-assisted single low-energy photon absorption process. Moreover, the results show that the AS PL can be used for all-optical high-temperature temperature sensing. Based on stable AS PL, a comparison of Stokes and AS excited ODMR signals as a function of laser power, MW power, and temperature was demonstrated, which both show similar behaviors. In addition, 
the AS excited ODMR contrast is several times larger than the Stokes excitation contrast, while the AS excited ODMR linewidth is smaller than the Stokes excitation one. The former might be explained by a resonant spin-flipping optical transition between the doublet metastable and excited state quartet, which implies the position of the doublet metastable level with respect to the ground state quartet at around $0.15 \mathrm{eV}$, in accordance with a recent ab initio study ${ }^{43}$. Furthermore, the AS excited ODMR signals are also more robust than Stokes excitation one at high temperatures, making it more suitable for quantum technologies at elevated temperatures. The measurement of Stokes and AS excited ODMR spectra at various magnetic fields shows that they have the same resonant frequency. On this basis, we realized the coherent control of defect spin under AS excitation at room temperature. The results demonstrate that the AS excitation methods not only preserve spin properties but also can improve the signal contrast around three times. Moreover, the AS excitation methods can also directly be used for other solid state qubits, including NV centers in diamond ${ }^{20}$ and divacancies ${ }^{8}$ and $\mathrm{NV}$ centers $\mathrm{s}^{25,26}$ in $\mathrm{SiC}$, etc. The experiments form a framework for developing AS excitation technologies in quantum information processing and quantum sensing.

\section{Methods}

Sample preparation. In the experiment, we use a high-purity $4 \mathrm{H}-\mathrm{SiC}$ epitaxy sample. Due to the low AS exciting efficiency, high-density $\mathrm{V}_{\mathrm{Si}}$ defect ensembles are required to obtain detectable signals. In view of this, we implanted $20 \mathrm{keV}$ helium ions $\left(\right.$ dose $1 \times 10^{13} / \mathrm{cm}^{2}$ ) and subsequently annealed the sample at $500{ }^{\circ} \mathrm{C}$ for $2 \mathrm{~h}$.

Experimental set-up. In the experiment, a home-made confocal system combined with a MW system is applied to excite and manipulate $\mathrm{V}_{\mathrm{Si}}$ defects. Two lasers with wavelengths of 720 and $1030 \mathrm{~nm}$, which are modulated by two separate acoustooptic modulators, are used to excite and polarize $\mathrm{V}_{\mathrm{Si}}$ defects, respectively. In order to efficiently collect $\mathrm{V}_{\mathrm{Si}}$ defect fluorescence, a non-polarizing beam splitter with 30/ 70 beam splitting ratio (Thorlabs, BS080) is used to separate the excitation and collection paths. For the room temperature ODMR and coherent spin control experiments, a 1.3 N.A. oil objective is used to efficiently collect the fluorescence. For the AS excited PL at low and high temperatures, a 0.65 N.A. air objective is used. In order to collect the $\mathrm{V}_{\mathrm{Si}}$ defect fluorescence under both Stokes and AS excitation, we simultaneously used an 850-nm long-pass filter and a $1000-\mathrm{nm}$ short-pass filter. The low-temperature experiment is performed using a Montana cryostation (4-350 K) combined with a confocal system. For the high-temperature experiments, the sample temperature is controlled and readout by a metal-ceramic heater (HT24S, Thorlabs) and a resistive temperature detector (TH100PT, Thorlabs), respectively. In the ODMR experiments, the fluorescence is collected by a multimode fiber into a femtowatt photoreceiver (OE-200-Si). For the readout of the ODMR signal, standard lock-in methods are used in the experiment, which is the same to the previous experiments $8,17,25,27$. The ODMR contrast is defined as $\triangle \mathrm{PL} /$ $\mathrm{PL}=V_{\text {Mod }} / V_{\text {Tot }}$, where $V_{\text {Mod }}$ is the magnitude of the $70 \mathrm{~Hz}$ modulated component of the output voltage and $V_{\text {Tot }}$ is the total time-averaged output voltage, which is the same with previous lock-in methods ${ }^{8}$.

\section{Data availability}

The data that support the findings of this study are available from the corresponding author upon reasonable request.

Received: 28 October 2020; Accepted: 30 April 2021;

Published online: 28 May 2021

\section{References}

1. Hensen, B. et al. Loophole-free bell inequality violation using electron spins separated by 1.3 kilometres. Nature 526, 682-686 (2015).

2. Kalb, N. et al. Entanglement distillation between solid-state quantum network nodes. Science 356, 928-932 (2017).

3. Humphreys, P. C. et al. Deterministic delivery of remote entanglement on a quantum network. Nature 558, 268-273 (2018).

4. Morioka, N. et al. Spin-controlled generation of indistinguishable and distinguishable photons from silicon vacancy centres in silicon carbide. Nat. Commun. 11, 2516 (2020).
5. Anderson, C. P. et al. Electrical and optical control of single spins integrated in scalable semiconductor devices. Science 366, 1225-1230 (2019).

6. Waldherr, G. et al. Quantum error correction in a solid-state hybrid spin register. Nature 506, 204-207 (2014).

7. Bradley, C. E. et al. A ten-qubit solid-state spin register with quantum memory up to one minute. Phys. Rev. X 9, 031045 (2019).

8. Koehl, W. F. et al. Room temperature coherent control of defect spin qubits in silicon carbide. Nature 479, 84-87 (2011).

9. Christle, D. J. et al. Isolated electron spins in silicon carbide with millisecond coherence times. Nat. Mater. 14, 160-163 (2015).

10. Widmann, M. et al. Coherent control of single spins in silicon carbide at room temperature. Nat. Mater. 14, 164-168 (2015).

11. Balasubramanian, G. et al. Nanoscale imaging magnetometry with diamond spins under ambient conditions. Nature 455, 648-651 (2008).

12. Dolde, F. et al. Electric-field sensing using single diamond spins. Nat. Phys. 7 , 459-463 (2011).

13. Kucsko, G. et al. Nanometre-scale thermometry in a living cell. Nature 500, 54-58 (2013).

14. Shi, F. Z. et al. Single-protein spin resonance spectroscopy under ambient conditions. Science 347, 1135-1138 (2015).

15. Simin, D. et al. All-optical dc nanotesla magnetometry using silicon vacancy fine structure in isotopically purified silicon carbide. Phys. Rev. X 6, 031014 (2016).

16. Wolfowicz, G., Whiteley, S. J. \& Awschalom, D. D. Electrometry by optical charge conversion of deep defects in 4H-SiC. Proc. Natl Acad. Sci. USA 115, 7879 (2018)

17. Zhou, Y. et al. Self-protected thermometry with infrared photons and defect spins in silicon carbide. Phys. Rev. Appl. 8, 044015 (2017).

18. Bourgeois, E. et al. Photoelectric detection of electron spin resonance of nitrogen-vacancy centres in diamond. Nat. Commun. 6, 8577 (2015).

19. Niethammer, M. et al. Coherent electrical readout of defect spins in silicon carbide by photo-ionization at ambient conditions. Nat. Commun. 10, 5569 (2019).

20. Tran, T. T. et al. Anti-Stokes excitation of solid-state quantum emitters for nanoscale thermometry. Sci. Adv. 5, eaav9180 (2019).

21. Wang, Q. X. et al. Photoluminescence upconversion by defects in hexagonal boron nitride. Nano Lett. 18, 6898-6905 (2018).

22. Wang, J. F. et al. Bright room temperature single photon source at telecom range in cubic silicon carbide. Nat. Commun. 9, 4106 (2018).

23. Lohrmann, A., Johnson, B. C., McCallum, J. C. \& Castelletto, S. A review on single photon sources in silicon carbide. Rep. Prog. Phys. 80, 034502 (2017).

24. Zargaleh, S. A. et al. Nitrogen vacancy center in cubic silicon carbide: a promising qubit in the $1.5 \mu \mathrm{m}$ spectral range for photonic quantum networks. Phys. Rev. B 98, 165203 (2018).

25. Wang, J. F. et al. Coherent control of nitrogen-vacancy center spins in silicon carbide at room temperature. Phys. Rev. Lett. 124, 223601 (2020).

26. $\mathrm{Mu}, \mathrm{Z}$. et al. Coherent manipulation with resonant excitation and single emitter creation of nitrogen vacancy centers in $4 \mathrm{H}$ silicon carbide. Nano Lett. 20, 6142-6147 (2020)

27. Wang, J. F. et al. On-demand generation of single silicon vacancy defects in silicon carbide. ACS Photon. 6, 1736-1743 (2019).

28. Gao, Y. F. et al. Phonon-assisted photoluminescence up-conversion of siliconvacancy centers in diamond. J. Phys. Chem. Lett. 9, 6656-6661 (2018).

29. Carter, S. G. et al. Spin coherence and echo modulation of the silicon vacancy in $4 \mathrm{H}-\mathrm{SiC}$ at room temperature. Phys. Rev. B 92, 161202(R) (2015).

30. Soykal, Ö. O., Dev, P. \& Economou, S. E. Silicon vacancy center in $4 \mathrm{H}-\mathrm{SiC}$ : electronic structure and spin-photon interfaces. Phys. Rev. B 93, 081207(R) (2016).

31. Dong, W. Z., Doherty, M. W. \& Economou, S. E. Spin polarization through intersystem crossing in the silicon vacancy of silicon carbide. Phys. Rev. B 99 184102 (2019).

32. Castelletto, $\mathrm{S}$. et al. Fluorescent color centers in laser ablated $4 \mathrm{H}-\mathrm{SiC}$ nanoparticles. Opt. Lett. 42, 1297-1300 (2017).

33. Barry, JohnF. et al. Sensitivity optimization for NV-diamond magnetometry. Rev. Mod. Phys. 92, 015004 (2020).

34. Abraham, J. B. S. et al. nanoTesla magnetometry with the silicon vacancy in silicon carbide. Preprint at https://arxiv.org/abs/2011.01137 (2020).

35. Nagy, R. et al. Quantum properties of dichroic silicon vacancies in silicon carbide. Phys. Rev. Appl. 9, 034022 (2018).

36. Yan, F. F. et al. Coherent control of defect spins in silicon carbide above $550 \mathrm{~K}$. Phys. Rev. Appl. 10, 044042 (2018).

37. Acosta, V. M. et al. Temperature dependence of the nitrogen-vacancy magnetic resonance in diamond. Phys. Rev. Lett. 104, 070801 (2010).

38. Toyli, D. M. et al. Measurement and control of single nitrogen-vacancy center spins above 600 K. Phys. Rev. X 2, 031001 (2012).

39. Anisimov, A. N. et al. Optical thermometry based on level anticrossing in silicon carbide. Sci. Rep. 6, 33301 (2016). 
40. Shang, Z. et al. Local vibrational modes of Si vacancy spin qubits in SiC. Phys. Rev. B 101, 144109 (2020).

41. Kraus, H. et al. Magnetic field and temperature sensing with atomic-scale spin defects in silicon carbide. Sci. Rep. 4, 5303 (2014).

42. Liu, G. Q. et al. Coherent quantum control of nitrogen-vacancy center spins near 1000 kelvin. Nat. Commun. 10, 1344 (2019).

43. Widmann, M. et al. Electrical charge state manipulation of single silicon vacancies in a silicon carbide quantum optoelectronic device. Nano Lett. 19, 7173-7180 (2019).

44. Nagy, R. et al. High-fidelity spin and optical control of single silicon-vacancy centres in silicon carbide. Nat. Commun. 10, 1954 (2019).

45. Udvarhelyi, P. et al. Vibronic states and their effect on the temperature and strain dependence of silicon-vacancy qubits in 4H-SiC. Phys. Rev. Appl. 13, 054017 (2020).

\section{Acknowledgements}

This work was supported by the National Key Research and Development Program of China (Grant Nos. 2016YFA0302700 and 2017YFA0304100), the National Natural Science Foundation of China (Grant Nos. U19A2075, 61725504, 61905233, 11975221, 11804330, 11821404, and 11774335), the Key Research Program of Frontier Sciences, Chinese Academy of Sciences (CAS) (Grant No. QYZDY-SSW-SLH003), Science Foundation of the CAS (No. ZDRW-XH-2019-1), Anhui Initiative in Quantum Information Technologies (AHY060300 and AHY020100), the Fundamental Research Funds for the Central Universities (Grant Nos. WK2030380017 and WK2470000026). A.G. acknowledges the support from the National Office of Research, Development and Innovation in Hungary for Quantum Technology Program (Grant No. 2017-1.2.1-NKP2017-00001), the National Excellence Program (Grant No. KKP129866), and the project Nanospin (EU QuantERA Grant No. 127902) as well as from the Ministry of Innovation and Technology for the Quantum Information National Laboratory. This work was partially carried out at the USTC center for Micro and Nanoscale Research and Fabrication.

\section{Author contributions}

J.-F.W. and J.-S.X. designed the experiments. J.-F.W. built the experiment set-up and performed the measurements with the help of F.-F.Y., Q.L., Z.-H.L., J.-M.C., and Z.-D.L. A.G. performed the theory modeling. J.-F.W., J.-S.X., and A.G. performed the data analysis and wrote the manuscript with contributions from all co-authors. J.-S.X., C.-F.L., and G.-C.G. supervised the project. All authors contributed to the discussion of the results.

\section{Competing interests}

The authors declare no competing interests.

\section{Additional information}

Supplementary information The online version contains supplementary material available at https://doi.org/10.1038/s41467-021-23471-8.

Correspondence and requests for materials should be addressed to A.G., J.-S.X. or C.-F.L.

Peer review information Nature Communications thanks the anonymous reviewers for their contribution to the peer review of this work.

Reprints and permission information is available at http://www.nature.com/reprints

Publisher's note Springer Nature remains neutral with regard to jurisdictional claims in published maps and institutional affiliations.

(c) (i) Open Access This article is licensed under a Creative Commons Attribution 4.0 International License, which permits use, sharing, adaptation, distribution and reproduction in any medium or format, as long as you give appropriate credit to the original author(s) and the source, provide a link to the Creative Commons license, and indicate if changes were made. The images or other third party material in this article are included in the article's Creative Commons license, unless indicated otherwise in a credit line to the material. If material is not included in the article's Creative Commons license and your intended use is not permitted by statutory regulation or exceeds the permitted use, you will need to obtain permission directly from the copyright holder. To view a copy of this license, visit http://creativecommons.org/ licenses/by/4.0/.

(C) The Author(s) 2021 